\title{
Report of the observations of AGL J1238+0406 (Fermi-LAT J123939+044409)
}

\author{
L. Pacciani ${ }^{a}$, I. Donnarumma ${ }^{a}$, G. Pucella ${ }^{b}$, E. Striani ${ }^{a}$, S. Vercellone ${ }^{m}$, V. Vittorini ${ }^{a}$, \\ M. Tavani ${ }^{a}$, A. Argan $^{a}$, G. Barbiellini ${ }^{i}$, A. Bulgarelli ${ }^{f}$, P. Caraveo ${ }^{e}$, M. Cardillo ${ }^{a}$,

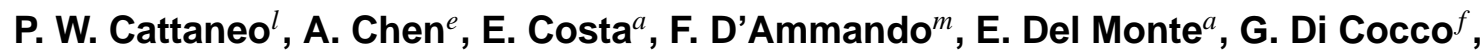

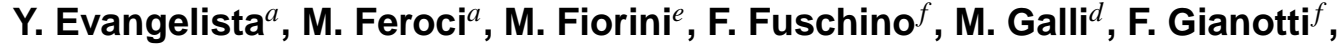 \\ A. Giuliani ${ }^{e}$, C. Labanti ${ }^{f}$, I. Lapshov ${ }^{a}$, P. Lipari ${ }^{n}$, F. Longo ${ }^{i}$, M. Marisaldi ${ }^{f}$, \\ S. Mereghetti ${ }^{i}$, E. Morelli ${ }^{f}$, E. Moretti ${ }^{i}$, A. Morselli ${ }^{c}$, A. Pellizzoni ${ }^{o}$, G. Piano ${ }^{a}$, \\ P. Picozza ${ }^{c}$, M. Pilia ${ }^{o}$, M. Rapisarda ${ }^{b}$, A. Rappoldi $^{l}$, A. Rubini ${ }^{a}$, S. Sabatini ${ }^{a}$,

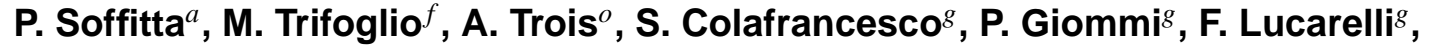 \\ C. Pittori $^{g}$, P. Santolamazza ${ }^{g}$, F. Verrecchia ${ }^{g}$, L. Salotti ${ }^{h}$ \\ ${ }^{a}$ INAF/IASF-Roma \\ via Fosso del Cavaliere, 100, I-00133 Roma, Italy \\ ${ }^{b}$ ENEA-Frascati \\ Via Enrico Fermi 45, I-00044 Frascati (Roma), Italy \\ ${ }^{c}$ Dip. Fisica, Univ. degli Studi di Roma, Tor Vergata \\ via della Ricerca Scientifica 1, I-00133, Roma, Italy \\ ${ }^{d}$ ENEA-Bologna \\ Via Martiri di Monte Sole, 4, I-40129 Bologna, Italy \\ ${ }^{e}$ INAF/IASF-Milano \\ Via E. Bassini, 15, I-20133 Milano, Italy \\ ${ }^{f}$ INAF/IASF-Bologna \\ via Gobetti, 101, I-40129 Bologna, Italy \\ ${ }^{g}$ ASI/ASDC \\ via Galileo Galilei, I-00044 Frascati (Roma), Italy \\ ${ }^{h}$ ASI \\ Viale Liegi, 26, I-00198, Roma, Italy \\ ${ }^{i}$ INFN Trieste \\ c/o Dip. Fisica - Univ. degli Studi di Trieste, Via Valerio, 2, I-34127 Trieste, Italy \\ ${ }^{l}$ INFN Pavia \\ Via Bassi, 6, I-27100 Pavia, Italy \\ ${ }^{m}$ INAF/IASF-Palermo \\ Via Ugo La Malfa, 153, I-90146, Palermo, Italy \\ ${ }^{n}$ INFN Roma I, \\ clo Dip. Fisica - Univ. degli Studi di Roma La Sapienza, P.le Aldo Moro, 2 - 00185 Roma - Italy \\ Italy \\ o INAF/Osservatorio Astronomico di Cagliari \\ Loc. Poggio dei Pini, Strada 54, I-09012 Capoterra (CA, )Italy \\ E-mail: luigi.pacciani@iasf-roma.inaf.it
}


AGILE pointed the Virgo Region for the first time for 3 weeks, starting from the second half of December 2007. We detected two FSRQ: 3C 273 [1] and 3C 279, and a unidentified gamma ray source: AGLJ 1238+0406. The source is positionally consistent with an AGN of the Sloan Digital Survey, namely SDSS J123932.75+044305 at $\mathrm{z}=1.76$. In the $2^{\text {nd }}$ edition of the Roma-BZCAT Multi-frequency Catalogue of Blazars [2] the source is associated with a Flat Spectrum Radio Quasar, BZQ 1239+0443. During the AGILE observation, INTEGRAL observed the same field for $600 \mathrm{ksec}$. IBIS did not detected the source in hard X-ray. OMC instead detected a weak signal from SDSS J123932.75+044305. AGL J1238+0406 has been already detected at low significance by EGRET [3], integrating the whole mission dataset. Then it was detected by FERMI one year after the AGILE pointing of the Virgo field. We report the simultaneous multiwavelength data obtained for the two periods of high gamma ray activity of the source.

25th Texas Symposium on Relativistic Astrophysics - TEXAS 2010

December 06-10, 2010

Heidelberg, Germany 


\section{The observations log}

AGILE [4] pointed the Virgo field the first time from 2007-12-16 17:20 to 2008-01-08 11:10. AGILE/GRID [5] detected the gamma source AGL J1238+0406 in very high state at the beginning of January 2008. INTEGRAL observed the same field simultaneously with AGILE for three orbits: from 2007-12-19 18:10 to 2007-12-22 06:40, from 2007-12-25 17:40 to 2007-12-28 06:30, and from 2007-12-31 17:10 to 2008-01-03 04:00. About one year after the AGILE detection, FERMI/LAT observed another high state activity period in gamma rays from a source positionally consistent with the AGILE source. The FERMI team reported a flux of $150 \cdot 10^{-8} \mathrm{ph} / \mathrm{cm}^{2} / \mathrm{s}$ (E > $100 \mathrm{MeV}$ ), integrating data of 2008-12-29 [6]. They obtained a photon index of $2.3 \pm 0.3$ for that integration. They localized the source with high precision, with an accuracy of 10 arcmin (Atel 1888). Following this accurate localization, the field was observed with Swift/XRT in X-rays starting from 2009-01-02 17:47, detecting an X-ray source with a flux of $6.2 \cdot 10^{-13} \mathrm{erg} / \mathrm{cm}^{2} / \mathrm{s}$. The KANATA $1.5 \mathrm{~m}$ telescope at Higashi-Hiroshima Observatory pointed the field. The optical observation revealed that the AGN SDSS J123932.75+044305 (at $\mathrm{z}=1.76$ ) was in high state, with V band magnitude of $15.7+/-0.1$ and $16.1+/-0.1$ at 2.8 and 3.8 (UT) January 2009 [7], to be compared with the archival magnitude of $20.60 \pm 0.02$ in $\mathrm{U}$ band.

The gamma ray source 3EG J1236+0457 [3] is positionally consistent with AGLJ 1238+0406. It was found in the EGRET data, integrating all the satellite observing periods. EGRET estimated a mean flux of $6 \cdot 10^{-8} \mathrm{ph} / \mathrm{cm}^{2} / \mathrm{s}(\mathrm{E}>100 \mathrm{MeV})$. Mattox et al. [8] already suggested the association of the SDSS source with the EGRET one, but the association could not be proven because the source has never been observed with higher flux in both optical and gamma ray.

\section{Analysis of data taken simultaneous with the AGILE detection of high gamma ray activity}

Level-1 AGILE-GRID data were analysed using the AGILE Standard Analysis Pipeline (BUILD20) and the AGILE Scientific Analysis Package [9], based on the likelihood method [10]. Albedo photons were rejected applying a cut of 85 deg centered on the Earth. We selected wellreconstructed gamma rays applying the FM3.119 filter, calibrated in the $100 \mathrm{MeV} \div 3 \mathrm{GeV}$ energy band. Counts, exposure and Galactic background gamma ray maps were created with bin-size of $0^{0} .1 \times 0^{0} .1$ for $\mathrm{E}>100 \mathrm{MeV}$.

Integrating the GRID data for 4 days between 2008-01-04 13:30 and 2008-01-08 11:10, we detected a source at $\mathrm{S} / \mathrm{NR}$ of $\sim 6$, located at $\mathrm{l}=296.22, \mathrm{~b}=67.14$, with an error circle of $(33+6)$ arcmin (statistical error at $95 \%$ confidence level and systematic respectively). The source is positionally consistent with SDSS J123932.75+044305. The flux was $\left(\sim 60 \cdot 10^{-8} \mathrm{ph} / \mathrm{cm}^{2} / \mathrm{s}(\mathrm{E}>100 \mathrm{MeV})\right.$ and the photon index $\sim 2$. The integration of the first week of observation with AGILE give no detection of sources at the position of SDSS 123932.75+044305, with an upper limit of $15 \cdot 10^{-8}$ $\mathrm{ph} / \mathrm{cm}^{2} / \mathrm{s}$. An image of the whole field, with a long integration not optimized for sources detection is reported in fig. 1.

Starting from the association of SDSS J123932.75+044305 with the gamma ray source [6], we are analysing the simultaneous data from OMC aboard INTEGRAL, searching the SDSS source in the OMC shots. From a preliminary analysis, a weak signal from SDSS J123932.75+044305 is 


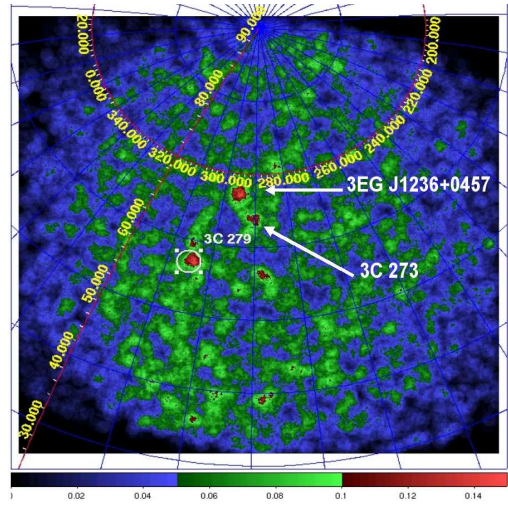

Figure 1: The 3 weeks integrated count map of the Virgo field obtained with AGILE from $100 \mathrm{MeV}$ to 3 $\mathrm{GeV}$.

detected for each INTEGRAL orbit, integrating all the shots inside it. The preliminary source flux is estimated at 17.5-16.5 magnitude in $\mathrm{V}$ band. This value is several magnitude brighter than the archival value in $U$ band. This fact gave us the confirmation of the association of the optical source with the gamma ray one. ISGRI did not detected the source neither on orbital (typically with 200ks of net exposure) time scale, nor from the integration of the data from the whole campaign (600 ks exposure). The third ISGRI observation was performed during the high activity period of the gamma ray source. From the third INTEGRAL observation, we derived a deep upper limit of $\sim 2$ mCrab (3 sigma U.L.) in 20-40 keV range.

\section{Further work on the source}

This distant blazar showed at least two bright gamma ray flares in the previous years. What is remarkable in the object is that the optical flux has raised of a factor $\sim 60$ with respect to the archival observation. The gamma ray flux raised of a factor $\geq 5$ for the flare detected by AGILE, and $\sim 10$ for the flare detected with FERMI.

The preliminary Spectral Energy Distribution for the two flaring activities detected by the two gamma ray observatories is reported in fig. 2 using almost simultaneous data.

We now have two broad band campaigns in the high state. To give a comprehensive picture of the object, we still need a number of informations: The black hole mass is still not known for the object. In fact it is usually inferred from the $\mathrm{H} \alpha$ and $\mathrm{H} \beta$ line width of the BLR, but this line cannot be observed in optical, due to the redshift of the source. We can overcome the problem making use of the CIV line width that is observable in optical band. It has recently proven [11] that CIV line width is correlated with the BH mass. A fundamental ingredient in the modeling of the spectral energy distribution is the contribution of seed photons to the external compton emission of the source. Ghisellini and collaborators [12] have parametrized those contribution starting from the knowledge of the disk luminosity. We will search in the archival data what we still miss, i.e. optical spectra and the photometry in the low state, to possibly reveal the disk luminosity of the source. From the modeling, we will estimate the jet power of the two flaring states. 


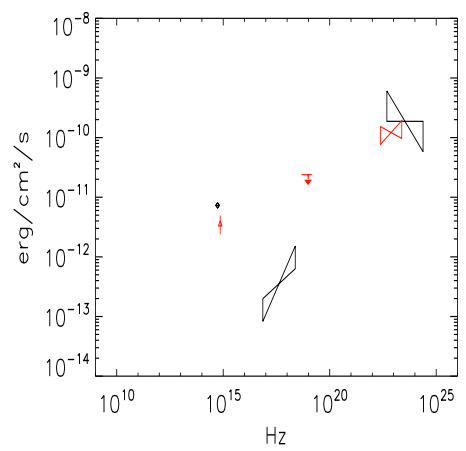

Figure 2: The simultaneous Spectral Energy Distribution for the two flaring episodes of AGL J1238+0406 (also known in gamma ray as 3EG J1236+0457, Fermi-LAT J123939+044409). Red data for observations simultaneous to AGILE. Black data for observations during the FERMI campaign.

\section{Acknowledgments}

We acknowledge financial contribution from the agreement ASI-INAF I/009/10/0.

The AGILE Mission is funded by the Italian Space Agency (through contract ASI I/089/06/2) with scientific and programmatic participation by the Italian Institute of Astrophysics (INAF) and the Italian Institute of Nuclear Physics (INFN).

\section{References}

[1] L. Pacciani et al, High energy variability of $3 C 273$ during the AGILE multiwavelength campaign of December 2007-January 2008, A\&A 494 (2009) 49-61;

[2] E. Massaro et al., 2010, The 2nd edition of the Roma-BZCAT Multi-frequency Catalogue of Blazars, ARXIV1006.0922;

[3] R. C. Hartman et al, The Third EGRET Catalog of High-Energy Gamma-Ray Sources, ApJS 123 (1999) 79-202;

[4] M. Tavani et al, The AGILE mission and its scientific instrument, Space Telescopes and Instrumentation II: Ultraviolet to Gamma Ray, Edited by M. J. L. Turner, G. Hasinger, Proceedings of the SPIE 6266 (2006) 626603;

[5] G. Barbielini et al, The Next Generation of High-Energy Gamma-ray Detectors for Satellites: The AGILE Silicon Tracker, Gamma 2001: Gamma-Ray Astrophysics, ed. S. Ritz, N. Gehrels, Chris R. Shrader, AIP Conf. Proc. 587 (2001) 754;

[6] A. Tramacere et al 2009, Fermi-LAT and Swift detection of a large GeV and optical flare from J123939+044409, Atel 1888, 2009;

[7] Y. Ikejiri et al, KANATA confirmation of the optical flare of SDSS J123932.75+044305, Atel 1892, 2009;

[8] J. R. Mattox et al, The identification of EGRET sources with Flat Spectrum Radio Sources, ApJ 481 (1997) 91-115;

[9] A. Chen et al, 2010, in preparation; 
[10] J. R. Mattox et al, The Likelihood Analysis of EGRET Data, ApJ 461 (1996) 396;

[11] R. J. Assef et al, Black Hole Mass Estimates Based on CIV are Consistent with Those Based on the Balmer Lines, ApJ submitted, arXiv:1009.1145;

[12] G. Ghisellini et al, Canonical high-power blazars, MNRAS 397 (2009) 985. 